\title{
Frontières entre littérature pour enfants et littérature pour adultes: Le Petit Prince comme modèle
}

\author{
Amal Abdel-Sattar Abd-Allah* \\ uni.aurore2@gmail.com
}

\section{Résumé}

Les frontières entre littérature pour enfants et littérature pour adultes sont reconnues de la part des hommes de lettres aussi que de la part des lecteurs. Il arrive parfois que ces frontières se confondent pour produire un genre mixte qui appartient apparemment à la littérature de l'enfance dans la forme, mais qui s'approche en quelque sorte à la littérature générale, dite des adultes, dans le fond.

Quant à son conte "Le petit prince", Saint-Exupéry en était aussi l'auteur des aquarelles qui illustrent le livre. L'écrivain lui-même est le narrateur du récit; il s'est inspiré de son métier d'aviateur certains faits dans le conte comme la panne de l'avion qui était le motif du récit et le début de sa rencontre avec le personnage principal, le petit prince. Cette partie répond à un véritable accident qui s'est passé à l'auteur au sein du Sahara en compagnie de son mécanicien aux années trente. Ce conte est adapté pour le cinéma en version animation par Mark Osbourne en 2015.

L'humanisme de cet ouvrage et la philosophie qui y abondent ne laisse aucun doute que le conte est destiné aux lecteurs de tous les âges. Partant de cette hypothèse nous allons analyser les différents aspects

* Maître de conférences à la faculté des langues (Al-Alsun) - Université de Minia.

(Frontières entre littérature pour enfants...) Dr. Amal Abdel-Sattar Abd-Allah 
qui font d'un tel ouvrage universel un chef d'œuvre pour les enfants et les adultes sur un pied d'égalité.

Mots-clés: littérature pour enfants, littérature pour adultes, Le petit prince, St. Exupéry.

\section{Introduction}

Ecrivain et aviateur, "il n'est pas essentiellement un homme de lettres, en particulier par la conception qu'il a de la littérature. Il est un homme d'action a qui l'action ne suffit pas". (BEAUMARCHAIS, Dictionnaire des littératures de langue française (S-Z), 1994, p. 2237). Antoine de St. Exupéry (19001944) est né dans une famille issue de la noblesse; son père est mort à l'âge de quarante et un ans; sa mère éprouvant un veuvage précoce le reste de sa vie a offert une bonne éducation à ses cinq enfants, dont Antoine auquel elle a transmis des valeurs humanistes qui influenceront l'écrivain toute sa vie. Antoine de St. Exupéry était connu comme écrivain, poète, aviateur et reporter français. Il est mort en 1944 pendant un vol de reconnaissance aérienne et on n'a jamais trouvé son corps. Bien qu'on ait trouvé l'épave de son avion en 2003, les circonstances de sa disparition sont inconnues. Malgré une vie très courte il a écrit des ouvrages renommés par son humanisme abondant dont Courrier sud, Vol de nuit, Terre des homme, Pilote de guerre et Le petit prince qui est traduit en plus de 300 langues. Ce dernier ouvrage était d'abord publié aux Etats-Unis un an avant sa mort et après son succès assourdissant il était publié en France en 1946 à titre

(Frontières entre littérature pour enfants...) Dr. Amal Abdel-Sattar Abd-Allah 
posthume. L'écrivain a reçu plusieurs prix littéraires soit en France, soit à l'étranger comme Femina pour Vol de nuit en 1931, le grand prix du roman de l'Académie française pour Terre des hommes en 1939 et National Book Award aux États-Unis en 1939.

Quant à son conte Le petit prince, Saint-Exupéry en était aussi l'auteur des aquarelles qui illustrent le livre. L'auteur en a déjà écrit deux versions: l'une en anglais en 1943 et l'autre en français en 1946, publiée en France deux ans après sa mort. L'écrivain luimême est le narrateur du récit; il s'est inspiré de son métier d'aviateur en faisant intervenir en faisant intervenir certains faits dans le conte comme la panne de l'avion qui était le moteur du récit et le début de sa rencontre avec le personnage principal, le petit prince. Cette partie répond à un véritable accident qui s'est passé à l'auteur au sein du Sahara en compagnie de son mécanicien aux années trente. Ce conte est adapté pour le cinéma en version animation par Mark Osbourne en 2015. (2021)

L'humanisme de cet ouvrage et la philosophie qui y abondent ne laissent aucun doute que le conte est destiné aux lecteurs de tous les âges. Partant de cette hypothèse nous allons analyser les différents aspects qui font d'un tel ouvrage universel un chef d'œuvre pour les enfants et les adultes sur un pied d'égalité.

C'est au cours des points essentiels, dans les pages suivantes, que nous allons montrer comment un livre classifié comme livre pour enfants pourrait dépasser la taxinomie qui lui est attribuée 
pour représenter un livre universel, pour l'humanité entière: à travers un aperçu général sur le conte, nous allons montrer les faits du conte et sa morale en guise de philosophie cachée, pas claire pour les lecteurs enfants, même pour un certain nombre de lecteurs adultes, mais avec la lecture attentive d'un lecteur vigilant et parfois critique la morale se révèlera peu à peu.

Les définitions déjà données à la littérature pour adultes ne les différencient pas d'une littérature pour enfants. Donc, les frontières entre les deux genres disparaissent dans certains points.

Aussi, la question de la littérarité n'éloigne pas encore l'écriture pour enfants $\mathrm{du}$ domaine. Ce qui lui rend sa considération.

La narratologie est la même, soit dans un roman pour adultes, soit dans un conte pour enfants. Par la suite, le sérieux s'y trouve et le lectorat en est le même.

Si la stylistique des deux types d'écriture est différente dans certains points, cela n'empêche pas le public adulte de consommer les écritures pour enfants, et pas le contraire. Parce que le contenu destiné aux enfants sera plus facile à assimiler que celui destiné aux adultes. Donc, le lectorat du contenu enfantin sera confondu. Ce qui fait du conte "Le petit prince" un livre littéraire général par excellence.

\section{La justification du choix}

Parmi les causes principales de notre choix du titre, l'ambiguïté de sa classification d'un conte considéré l'une des figures 
classiques de la littérature d'enfance dans le monde entier. Le fait de sa traduction envers plus de 300 langues est la preuve de sa notoriété en tant que conte pour enfants. Mais le fait de sa lecture approfondie est la cause du paradoxe de sa classification. La philosophie du livre, la sagesse cachée entre les lignes et le message humaniste enfoui dans ses feuilles nous ont encouragé à reconsidérer le conte du point de vue de sa taxinomie.

\section{Problématique de la recherche}

La question qui se pose à propos de cette approche est la suivante: le conte du Petit prince, peut-il sortir de sa classification traditionnelle comme un conte de littérature pour enfants, pour représenter un conte de littérature pour adultes; ou, peut-il figurer un genre métisse réunissant les deux? Et, quels sont les procédés dont a disposé l'auteur pour achever ce mélange, s'il en avait déjà l'intention?

\section{1. "Le Petit prince": faits et morale}

L'écrivain, Antoine de St-Exupéry, s'est inspiré de son métier d'aviateur plusieurs ouvrages dont Le Petit prince en sont l'exemple. Racontée à la première personne, l'histoire nous introduit à une scène désertique où l'avion du narrateur, qu'il en est le pilote lui-même, vient de tomber en panne en plein désert du Sahara. Cet accident lui a offert une chance d'or: la rencontre du petit prince. Ce dernier était un petit bonhomme à la chevelue dorée, venu d'une autre planète trop petite appelée l'astéroïde B 612. Au cours de son voyage, le petit prince avait visité plusieurs 
planètes, dans lesquelles il a rencontré divers hommes ayant chacun un travail à faire, et dont les rencontres reflètent des analogies avec le monde matériel de la planète de la Terre. La prélude du voyage avait commencé plutôt avec un souvenir d'enfance de l'écrivain narrateur qui était, à cause des adultes, dévié d'une carrière qu'il a souhaitée: quand il était à l'âge de six ans il avait dessiné un éléphant avalé par un serpent boa; un dessin dont on ne voit que la bosse créée dans le ventre du serpent. Les adultes prenaient son croquis pour un chapeau, et bien qu'il ait expliqué par un deuxième dessin les détails du premier, les adultes l'ont conseillé de laisser la peinture pour étudier les mathématiques et la géographie; un conseil qui lui a coûté une carrière artistique à abandonner. La société nous juge, la plupart du temps, elle nous contraint de faire ce qu'elle voit convenable. Donc, nous n'avons pas le droit de choisir ce que nous aimons, mais ce que l'entourage voit et préfère.

Le détour dans la vie du narrateur était quand il est devenu aviateur grâce au conseil qu'il a reçu à son enfance. Il a vraiment oublié ce qu'il était en tant qu'enfant, mais le petit prince est venu pour le lui rappeler.

Le petit prince est un personnage curieux qui n'abandonne aucune question sans y recevoir une réponse. Au long de son parcours il interroge les personnages qu'il rencontre sur la raison de leurs actions afin de comprendre leurs comportements. Cela 
donne au prince la sagesse que le narrateur adulte n'a jamais récupérée durant sa vie.

Dans la première planète, le petit prince a rencontré un souverain dont les commandements étaient parfois irraisonnables. Il n'y avait personne à régir ou commander, donc c'était illogique de donner des ordres. L'autorité ne serait pas respectée sans logique ou raison. A la suite du roi, dans le deuxième voyage, le petit prince avait rencontré le vaniteux: ce personnage habitant une planète où personne d'autre ne s'y trouve, ne fait rien d'utile, mais il attend qu'on l'applaudisse pour toute action. La vanité et l'égocentrisme ne sont autre chose qu'absurdes, notamment lorsqu'ils caractérisent les personnes inutiles.

Le buveur est un autre personnage qui habitent la planète suivante. Dans une action absurde et paradoxale, il boit pour oublier qu'il a honte de boire. Un personnage indécis qui tombe dans le piège de rien faire pour échapper à ce cercle vicieux.

La visite suivante sera au businessman, un homme occupé de compter les étoiles qu'il prétend posséder! L'approche est claire quand il s'agit de l'argent. Le message est également clair quand il s'agit de l'inutilité d'une chose qui n'apporte pas de valeur à la personne qui la possède. A l'encontre du roi qui suppliait le petit prince pour rester chez lui, en lui offrant le poste du ministre, à moins qu'il trouve un sujet pour régner, le businessman n'apprécie pas l'existence du petit prince parce qu'il était occupé de compter les étoiles. Ensuite, le petit prince arrive à une petite planète ou 
l'allumeur de réverbères y existe tout seul. Il allume et éteint son réverbère selon la consigne. Au moins, le petit prince trouve que son travail est utile, mais son respect de la consigne le rend fatigué car il n'a pas le temps de se reposer. Sur la planète suivante le petit prince rencontre le géographe qui dépend des travaux des explorateurs. Ces derniers agissent véritablement; ils voyagent et relatent ce qu'ils voient, tandis que le géographe dessine seulement les cartes. C'est un travail utile mais beaucoup dépendant du travail des autres.

La terre est la dernière destination du petit prince. Il y rencontre l'aiguilleur, ce personnage qui envoie les trains vers des destinations inconnues. Le petit prince lui demande sur ce que cherchent ces gens-là, mais l'aiguilleur lui répond que même le chauffeur de locomotive ne le sait pas! C'est important de connaitre sa destination, et par la suite son but. La vie n'aura pas de sens si l'on n'a pas de but à réaliser.

Plusieurs reprises dans le conte nous montrent la beauté et la simplicité de la planète du petit prince: il se doit de temps en temps arracher les mauvaises herbes et soigner les bonnes herbes. Il doit distinguer, dès le début, les bonnes des mauvaises herbes, de peur que les baobabs, passant d'abord par la phase de brindilles, grandissent et détruisent sa plus petite planète par ses racines. Le petit bonhomme doit aussi ramoner les trois volcans qui résident dans sa planète, afin d'éviter leur éruption qui détruirait aussi sa planète. Ces actions rappellent celles du 
ménage: la petitesse de la planète du petit prince la renvoie à une maison. Le soin des herbes est comme le jardinage, le ramonage des volcans est pareil au nettoyage des cheminées, etc.

Le côté émotionnel nous est évident: du fait que le petit prince soigne la seule créature aimée dans sa planète, qui est la rose, il serait important de signaler que cet amour était inconditionnel. Il la met sous globe, il la protège du vent et l'arrose régulièrement.

Le conte est plein d'émotions aussi entre les hommes qu'entre les différentes espèces: l'amour et l'amitié étaient déjà entre le petit prince et la rose, le petit prince et le renard et enfin entre le petit prince et l'aviateur. Ce dernier nouait une sorte d'amitié ou de fraternité qui unirait deux êtres de deux planètes différentes. La première rencontre dans le désert était miraculeuse: un aviateur dont l'avion était en panne avec un enfant à la chevelure dorée en plein désert. Comment peut-on imaginer la surprise d'un adulte au désert sollicité par un enfant de lui dessiner un mouton? Quant à l'aviateur, les quelques premiers croquis du mouton réclamés du petit prince ne suffisent pas à satisfaire l'ambition du petit, qui, même après lui montrer les deux anciens dessins de l'aviateur, avait refusé qu'on lui offre un serpent boa avalant un éléphant; ce qui stupéfiait l'aviateur! Donc, il lui fallait enfin lui dessiner une caisse avec des trous en lui expliquant que le mouton s'y trouve, et que les trous sont pour lui permettre de respirer! Le petit prince accepte avec plaisir cette explication et remercie l'aviateur qui, 
étonné, ne pouvait pas mesurer le charme de l'imagination enfantine qui permettrait l'impossible de se passer.

L'amitié se fortifiait dorénavant entre l'aviateur et le petit prince: ce dernier lui a raconté ce qu'il a rencontré durant son voyage de son astre à la terre. Toutes les amitiés qu'il a faites avec la fleur, le renard, et aussi l'aviateur, nous donnent beaucoup de leçons sur la fidélité et l'abnégation du petit bonhomme.

Avant la rencontre du petit prince avec le renard, il a vu un jardin plein de plusieurs sortes de fleurs, ce qui lui prouve que la fleur unique qu'il a laissée dans sa planète n'était pas unique au monde comme il a cru. Cette vérité l'attristait profondément et il a pleuré. Après sa rencontre avec le renard sur la planète de la terre, ce dernier lui a enseigné le sens du mot "apprivoisement": c'est en créant des liens avec les autres, et ensuite en découvrant qu'un seul lien perdurerait si l'on partage des choses ou des souvenirs ensemble, que ce lien serait unique au monde.

La scène de clôture du conte est en quelque sorte tragique: le serpent, la créature méchante, était la clé de délivrance du petit prince. Une morsure lui a permis de quitter la terre pour retourner à sa planète afin de rencontrer sa rose aimée, la seule créature vivante à laquelle il s'intéresse sur sa planète. Les couchers du soleil lui manquent aussi. L'adieu était si difficile pour l'aviateur qui n'a pas pu supporter le départ du petit prince. Ce dernier, pour le consoler, lui a conseillé de lever ses yeux vers les étoiles pour imaginer entendre les rires du petit prince. Ce faisant, toutes les 
étoiles seront des grelots pour l'aviateur, alors que pour d'autres elles seront des lumières dans le ciel.

\section{Définitions et frontières}

La problématique de la définition semble être d'une telle polémique, non pas seulement pour la difficulté de distinguer les aspects marquant littérature pour enfants de littérature pour adultes, mais aussi pour la difficulté de cerner le public visé des deux écritures qui se confondent la plupart du temps en faisant cette confusion. Louise Daubigny s'interroge sur la problématique de la dénomination de ce genre littéraire selon l'âge même du destinataire: " Le critère de l'âge est moins subjectif que les critères sociologiques. Très vite, cependant, on a vu s'élever le soupçon que, derrière cette dénomination apparemment objective, se cachait un jugement de valeur dépréciatif : n'est-il pas logique de penser que la littérature pour enfants, vu le public auquel elle s'adresse, est moins complexe, moins libre, moins riche - et, donc, de moindre qualité que la littérature que l'on qualifiera par commodité de « générale » ?" (JOUVE, 2002)

Plusieurs nominations se confondent en cette matière: livre pour enfants, livre de jeunesse, livre pour l'enfance et la jeunesse, etc... Toutes ces nominations "suggèrent un livre destiné par sa forme et son contenu à l'enfance: à l'enfant lecteur... Il est nécessaire de distinguer livre pour enfants et littérature pour la jeunesse, même si cette distinction reste somme toute théorique. Si on s'intéresse aux publications destinées aux enfants dans une 
perspective littéraire, on parlera de littérature pour la jeunesse. $\mathrm{Si}$ on adopte une perspective historique, en considérant tous les ouvrages écrits et édités pour les enfants- abécédaires, manuels scolaires, documentaires, albums, romans, contes, revues, ... on parlera de livres pour enfants". (SAYED, 2018, p. 34) Nous nous intéressons, dans notre présente recherche, au mélange entre les deux. Ce mélange visant le lecteur enfant par le contenu luimême, et en même temps soucieux de montrer l'éthique dans cette pratique littéraire.

\section{Question de littérarité}

La problématique de la définition de ce genre entraîne une question de soupçon sur sa littérarité. A ce propos, Vincent Jouve reprend son apologie en disant: 'Les écrivains pour enfants, sans doute en quête de considération (mais en quoi s'adresser à l'enfance est-il déshonorant?), tendent à répondre par l'affirmative. Leur pratique, disent-ils, n'est pas fondamentalement différente de celle des autres écrivains : non qu'écrire pour les enfants n'impose certaines contraintes, mais seul l'emballage change ; les caractéristiques profondes - celles qui définissent un texte comme esthétique - restent celles de la littérature générale". (JOUVE, 2002, p. 70)

A cet égard, notons que 'La littérature adressée à l'enfance ne s'est jamais située en dehors de la littérature que lisent les adultes. Elle se porte seulement vers des lecteurs qui n'ont pas les mêmes interrogations sur le sens du monde que leurs parents, qui n'ont 
pas non plus la même expérience de la langue [...] En effet, nous touchons là un paradoxe propre à la littérature de jeunesse: le public lecteur n'achète pas lui-même les ouvrages qu'il lit. Il opère ses choix à partir des prescriptions de ses parents, de ses enseignements, ou de ses goûts personnels.". (SAYED, 2018, p. 22)

La littérarité d'un genre se montrerait à travers des indices qui illuminent le récepteur, soit le lecteur en général, sur l'objectif de l'écriture de cet ouvrage, et par la suite sa portée morale. C'est en passant par certaines étapes qu'on parvient à l'image totalitaire de l'ouvrage supposé littéraire. Ces étapes, ou dimensions, s'entremêlent entre elles afin de donner à l'ouvrage sa valeur littéraire, et par conséquent il n'est pas important si le lecteur est enfant ou adulte, parce que la question dépasserait le problème du public visé à celui de la valeur artistique attribuée à l'ouvrage luimême.

\section{a.Dimension narratologique}

Vincent Jouve s'accorde avec Greimas dans sa vision montrant que "tout récit est une quête mettant en scène un sujet à la recherche d'un objet; on rencontrera nécessairement, quelle que soit la nature de l'objet recherché (le sens de la vie ou la guérison du petit frère, la pierre philosophale ou l'amour des parents), des personnages contrariant la réalisation du projet (les opposants) et d'autres la favorisant (les adjuvants). Que toute quête ait une 
origine (le destinateur) et des bénéficiaires (les destinataires) va également de soi". (JOUVE, 2002, p. 71)

Le conte pour enfants ne diffère en aucun point technique du roman classique. À ce propos, on trouve dans les deux que "[les] jeux sur le statut du narrateur (présent ou absent de l'histoire), sur les points de vue (les exemples de focalisation interne ne sont pas moins rares dans les récits pour la jeunesse que dans les textes pour adultes) ou sur l'ordre de la narration (Le Petit Prince, déjà, s'ouvrait sur l'enfance du narrateur)" (JOUVE, 2002, p. 71), tous ces éléments s'y trouvent sur un pied d'égalité.

L'opération de la lecture s'avère de plus en plus compliquée à cause de plusieurs pratiques de la part du lecteur. Influencé par la division de Michel Picard, Jouve voit qu'on repère chez le lecteur adulte trois instances essentielles qu'il appelle le liseur, le $l u$ et le lectant: " Le liseur est défini comme la part du sujet qui, tenant le livre entre ses mains, maintient le contact avec le réel extérieur ; le lu comme l'inconscient du lecteur réagissant aux structures fantasmatiques du texte ; et le lectant comme l'instance de la secondarité critique qui s'intéresse à la complexité de l'écrit. Or, si les deux premières instances jouent un rôle essentiel dans la lecture enfantine, la troisième semble moins sollicitée" (JOUVE, 2002, p. 76). Pour le lecteur enfant, il y a vraiment du liseur: ce côté est plus important pour l'enfant que pour l'adulte; dans la mesure où la pratique même de lecture le lie directement avec le réel (siège, dossier, table, ...etc.); ce qui fait enfin que 
l'expérience ne lui sera pas oubliée. L'instance du $l u$ n'est pas moins importante pour le jeune lecteur: l'inconscient est d'autant plus existant chez un enfant dont l'imaginaire est frais et prédominant. Au contraire, le côté lectant est moins requis chez l'enfant que chez l'adulte: la vision critique est une fonction du savoir, de la culture et de la maturité; ce qui mène le lecteur expérimenté à s'intéresser plus au signifié qu'au référent. Ce sera, par conséquent, impossible de solliciter à l'enfant une action pareille. Par rapport à ce dernier point, il est à signaler que les textes destinés aux adultes acceptent plusieurs interprétations, ce qu'on inscrit dans un acte de pluralité de significations; tandis que le texte pour enfants est plus monosémique et moins pluriel. (JOUVE, 2002, pp. 76-77)

Signalons aussi que la lecture d'un texte pour enfants de la part d'un adulte n'empêche pas ce processus d'interprétation: l'adulte pourrait trouver du sens profond qui n'est pas assimilable par un enfant. Et c'est apparemment le cas du Petit Prince.

\section{b. Dimension stylistique}

Dominique Demers se contente de constater que la littérature pour enfants n'est pas une littérature autre, mais une littérature plus. (DEMERS, 1998, pp. 28-30) Les différences, entre les deux littératures ${ }^{1}$, s'avèrent d'abord quantitatives: 'Écrire pour la jeunesse, c'est donc d'abord écrire tout court.[...] Une œuvre brève n'est pas nécessairement simple". (DEMERS, 1998, p. 28)

1 On a déjà déduit qu'il s'agit aussi de la littérature, pas moins importante.

(Frontières entre littérature pour enfants...) Dr. Amal Abdel-Sattar Abd-Allah 
Si nous tenons à nous plonger dans l'univers stylistique de cet ouvrage nous voyons que la forme même semble différente et unique: l'ouvrage est un conte à écriture pure et simple et à vocabulaire aisé. Les dialogues, la narration à la première personne et même les dessins simples, faits par l'auteur lui-même, tout cela nous donne cette image complète d'un conte pour enfants.

\section{- La dédicace}

$\mathrm{Si}$ nous avons à nous approfondir un peu plus pour lire les composants de ce conte simple et clair nous allons découvrir dans la dédicace une dimension plus profonde qu'elle nous semble. Saint-Exupéry y a écrit:

« À Léon Werth.

Je demande pardon aux enfants d'avoir dédié ce livre à une grande personne. J'ai une excuse sérieuse : cette grande personne est le meilleur ami que j'ai au monde. J'ai une autre excuse : cette grande personne peut tout comprendre, même les livres pour enfants. J'ai une troisième excuse : cette grande personne habite la France où elle a faim et froid. Elle a besoin d'être consolée. Si toutes ces excuses ne suffisent pas, je veux bien dédier ce livre à l'enfant qu'a été autrefois cette grande personne. Toutes les grandes personnes ont d'abord été des enfants. (Mais peu d'entre elles s'en souviennent.) Je corrige donc ma dédicace :

À Léon Werth quand il était petit garçon » (EXUPERY, 2003, p. 7)

(Frontières entre littérature pour enfants...) Dr. Amal Abdel-Sattar Abd-Allah 
Cette introduction géniale ouvre la voie devant un ouvrage apparemment enfantin mais profondément il porte en lui des germes plus mûrs, susceptible enfin d'être lu par les jeunes aussi que les adultes. Entre les lignes de l'ouvrage, dans le sens des dialogues et des réflexions du héros, et même de l'auteur, on signale aussi une sagesse cachée, pareille à celle de la dédicace, qui donne une pesanteur et un poids philosophique au conte. Les phrases simples et courtes donnent l'apparence aux proverbes, ou maximes; ces allocutions qui portent la sagesse populaire et universelle.

\section{- La répétition}

La plupart du temps, l'écrivain a recours à la reprise de certaines phrases à la manière des contes de fées, ou les histoires de Mille et une nuits. L'apparence narratives de ces propos les implique dans la pratique littéraire des contes pour enfants, mais n'empêche pas qu'on s'enlise dans un exercice littéraire au sens général si l'on parle d'une littérature pour adultes. Par exemple, nous rencontrons cette formule plusieurs fois: "à mille milles de toute région habitée" (EXUPERY, 2003, pp. 11-12). Cette formule introduit au lecteur, soit enfant ou adulte, l'image du désert si loin de tout aspect de vie. La répétition même donne l'influence de l'écho qui se reprend plusieurs fois dans le vide; ce qui baigne le lecteur totalement dans un sentiment comme s'il se trouve seul et exilé dans un 
espace désertique. Un autre exemple nous affirme l'insistance de la part de l'écrivain à adopter cette technique de narration spécifique à l'écriture enfantine: "le petit prince, qui jamais de sa vie n'avait renoncé à une question, une fois qu'il l'avait posée" (EXUPERY, 2003, pp. 27, 45, 54, ...). Cela montre la ténacité du petit prince qui voulait tout savoir et ne se retire avant de trouver des réponses aux questions qu'il pose constamment. La répétition, comme un style poétique connu, est très rare dans la prose, si elle ne délivre pas un certain message, qui est en ce cas donner la forme traditionnelle des contes pour enfants. Mais la répétition dépasse, chez St. Exupéry, le but formel pour donner une dimension sérieuse.

\section{- La longueur des chapitres}

La longueur des chapitres se diffère selon le message que l'auteur voulait donner. A la manière d'un enfant, il dit ce qu'il veut dire sans ornement. Une fois le message dit, il met un point pour terminer une partie de son conte, et c'est simplement accepté de la part du lecteur plus âgé. L'important est dit, pas besoin, donc, de charger les lignes avec des images figuratives ou un style sophistiqué. Chaque chapitre contient seulement des dialogues, à l'exception de quelques explications en de courtes lignes. Pas beaucoup de détails, pas beaucoup d'artifice, le message du petit prince va "droit au cœur du lecteur". (BORGI, 2020, p. 9) 


\section{- Le choix des personnages}

Si nous tenons aussi à nous plonger dans le sens de ce conte, nous allons découvrir différentes procédures que l'écrivain s'en servait volontiers pour mettre en relief son message.

Le petit prince, dans son voyage pour découvrir le monde, était entouré de plusieurs personnages. Ceux-ci, soient principaux ou secondaires, avaient certains rôles qui mènent, chacun, le lecteur à voir une partie de l'image avant qu'elle soit complète en fin de compte.

Les personnages principaux dans le conte, à part le petit prince, sont la rose, le renard et le serpent. Chacun présente un poids de symbole qui pèse tout au long de l'histoire: la rose, en relation énigmatique avec le petit prince, symbolise la femme avec toutes ses contradictions. Une gamme d'attributs féminins lui est accordée; le lecteur peut simplement découvrir.(pp. 30-36). Rouba Borgi décide cette fois de faire une ressemblance entre ce lien et celui qui lie Adam avec Ève: "Enfin, c'est à cause de la rose, un peu à cause de son mensonge, que le petit prince a décidé, malgré lui, de quitter sa planète; c'est pareil à Adam qui, à cause d'Eve, et surtout de son mensonge, a été obligé de quitter le paradis: première ressemblance avec un symbole biblique". (BORGI, 2020, p. 11)

Le serpent était le premier personnage que le petit prince rencontre sur la terre. Il était aussi le dernier. Le symbole du serpent est évident: comme un signe du mal et du diable, le long 
de sa rencontre avec le petit prince on attendrait une sorte d'acte malicieux ou de trahison de la part du serpent. Ce qui s'est passé était le contraire: même que le serpent s'est lové autour de la cheville du petit prince, mais il ne l'a pas touché grâce à son innocence et sa pureté. $^{2}$ La deuxième et dernière rencontre était décisive dans la vie du petit prince: même si le serpent l'a mordu cette fois, mais il semble que c'était comme un dernier vou du petit prince, déjà ennuyé de sa présence sur la terre et résolu de la quitter pour retourner à sa planète, à sa belle rose, et à ses beaux volcans. (EXUPERY, 2003, pp. 83-84). Le vocabulaire employé par l'auteur ne criminalise jamais le serpent: aucun mot utilisé pour décrire l'acte du serpent comme le vocabulaire ordinaire. Il n'y a pas de "mordre" ni de "tuer" ou "exécuter". Il semble, selon Borgi, que si dans 'l'inconscient collectif, le serpent symbolise Eve; l'auteur voulait-il se réconcilier avec la Femme qui, représentée par la rose, a chassé le petit prince de son paradis, alors, il a fait de sorte que c'est une femme - Eve, le serpent - qui l'aidera à y retourner? [...] En fait, dans la Bible il y a deux serpents: celui qui tente Eve et un autre d'airain qui sauve celui

\footnotetext{
${ }^{2}$ La première rencontre du petit prince avec le serpent au cours de sa visite de la planète de la Terre n'était pas tragique, comme I'on imagine avec ce type de rencontres fatales; elletémoigne d'une conversation très familière entre les deux: "

- Tu es un drôle de bête, mince comme un doigt.

- Mais jesuis plus puissant que le doigt d'un roi. [...]

- Tu n'es pas bien puissant... tu n'as même pas de pattes... tu ne peux même pas voyager.

- Je puis t'emporter plus loin qu'un navire. Il s'enroula autour de cheville du petit prince, comme un bracelet d'or: Celui que je touche, je le rends à la terre d'où il est sorti [...] Mais tu es pur et tu viens d'une étoile. " (p.60).
}

(Frontières entre littérature pour enfants...) Dr. Amal Abdel-Sattar Abd-Allah 
qui le regarde ( (Nombres, 2021). Le serpent, "anneau couleur de lune" ( (EXUPERY, Le petit prince, 2003, p. 59), pareil au serpent biblique du métal, a sauvé le petit prince." (BORGI, 2020, p. 12). Les bons caractères accordés faussement à cette créature ne sont pas suffisantes pour renvoyer une image paradoxale: le serpent semble un personnage sage qui parle fréquemment par énigmes. (EXUPERY, 2003, p. 62)

Le renard, un autre personnage important dans le conte. Porteur de caractères qu'il partage aussi avec l'homme comme la ruse et l'astuce, il semble aussi chercheur d'une trouvaille que tout le monde cherche et que ne trouvent que les chanceux: l'amitié. Ce dernier mot est déjà signalé de la part du renard par un autre mot qu'il apprend du petit prince, qui est "l'apprivoisement". C'est un terme chargé de plusieurs connotations dont le domptement et le contrôle d'un animal, mais qui acquiert en ce cas une dimension plus hautaine, dont l'amour et le partage sont des sens qui unissent l'être humain avec l'animal. Le dialogue fait par St. Exupéry dans son conte est très intéressant: c'est par de simples paroles que l'auteur traduit aux enfants un sens un peu vague pour eux, dont ils ne connaissent que la nomination. En même temps, il montre aux adultes un angle différent du sens de l'amitié, un sens plus profond que ce qui nous semble. (EXUPERY, 2003, pp. 67-69). 


\section{c. Dimension esthétique}

Dans cet ordre, la beauté de l'ouvrage littéraire s'aperçoit au cours des aspects cachés qu'un lecteur érudit peut découvrir plus ou moins savamment chacun selon son sens. Dans les pages suivantes nous allons pister les signes de cet aspect esthétique:

\section{- Symbolisme}

La dimension symbolique du conte se dévoile, peu à peu, à travers un œil vigilant, qui n'est autre que celui d'un lecteur adulte. Si le regard enfantin ne dépasse pas les illustrations claires, le vocabulaire simple et les images superficielles, celui de l'adulte ne les prend pas à la légère: " Toutes les visites du petit prince parviennent ainsi à ébranler le pouvoir autoritaire d'un roi, la prétention d'un vaniteux, l'échec d'un buveur, la stérilité du métier du businessman, la science morte d'un géographe, l'aliénation d'un allumeur de réverbères ou l'agitation d'une planète terre." (MOURIER, 2001, p. 47)

Tous les personnages que le petit prince avait rencontrés au cours de son aventure ne sont que symboles révélateurs d'un sens plus loin de la vision simple et directe de l'enfant lecteur. Même la réalité de la mort, en étant un dosage lourd à digérer de la part de l'enfant, l'auteur en donne des indications qui ne sont perceptibles que par un lecteur "en état d'alerte, réceptif à la gravité du message livré". (MOURIER, 2001, p. 47). Le retour du petit prince à sa planète lui exige une mort réelle sur la terre: la morsure du serpent, la pâleur du petit prince, la peur et la 
souffrance, tout cela se réalise par un vocabulaire consolant, apaisant la gravité de la situation pour un récepteur mineur. On a besoin d'atténuer la vérité affligeante de la mort; c'est pour cela le départ du petit prince était solennellement symbolisé par la disparition après avoir quitté ses vêtements. Cette disparition n'est qu'une mort abstraite: " la mort peut être celle de l'enfance, elle consacre la naissance à la responsabilité. Tel un serpent muant, le petit prince quitte son vêtement d'enfant et endosse celui du sage [...] ". (MOURIER, 2001, p. 49) Et, la mort mène à la renaissance, le passage de l'innocence à la responsabilité: " Cette naissance mystique n'est-elle pas emblématique d'une maturation spirituelle ? « Tu sais... ma fleur... j'en suis responsable! »". (MOURIER, 2001, p. 49) Les sept planètes visitées par le petit prince, y incluse la Terre, nous rappellent les maux qui ont frappé la société moderne. Ces visites symbolisent "la déception qui est née d'une découverte de l'égoïsme, du matérialisme, de la vanité, de l'aliénation. Tous retranchés derrière des objets ou amoureux des chiffres, des records à battre ou à posséder, leurs habitants occultent l'angoisse, évitent d'être pour mieux paraître." (MOURIER, 2001, p. 50). C'est tout ce qu'on peut trouver de matérialité dans une communauté qui cultive cinq mille roses dans un même jardin sans y trouver ce qu'elle cherche, ni bonheur, ni beauté, mais pur divertissement. A ce propos, Mourier développe une explication intéressante des attitudes des habitants des planètes visitées par le petit prince: à part la déshumanisation 
à laquelle s'entrainent les personnages, leur attitude témoigne d'une sorte d'" incarnation d'un pouvoir totalitaire qui réduit tout homme à l'état de sujet, le bel orateur de roi veut en faire un adepte. Culte de la personnalité du vaniteux ou possession par le vice du buveur, possession par la soif de possession du businessman ou possession par un système ou un métier de l'allumeur ou du géographe..., leur rôle les rend tous esclaves. Ainsi la possession devient-elle une terrifiante normalité comme celle d'être rhinocéros chez Ionesco. Cette possession devient infernale en donnant l'illusion d'être. [...] Ce détournement des fonctions spirituelles les mène parfois au refus de la vie concrète et des actions comme dormir, boire, même au risque de leur équilibre comme le prouvent l'allumeur de réverbères, privé de sommeil par fidélité aveugle à la consigne, et le marchand de pilules perfectionnées qui apaisent la soif. Les grandes personnes voient leur conscience captive et ressemblent souvent à des mythomanes, des masochistes ou encore des êtres absurdes peu à peu gagnés par la folie. L'absence de sentiments, sorte d'anémie d'amour, les contraint à la possession par le vide. Le plus tragique est probablement la perte de conscience de leur souffrance, pourtant visible sur les aquarelles de l'œuvre." (MOURIER, 2001, p. 50) .

Le conte est surchargé de symboles plus ou moins évidents aux yeux du lecteur adulte conscient du message humaniste que l'écrivain voudrait délivrer. D'une part, les personnages 
représentent une diversité de caractères humains résidant à l'intérieur de chacun de nous: le renard, le marchand de pilules, l'aiguilleur, le businessman, ...tous ces personnages renvoient à des caractères humains réels, matérialistes, frivoles, avides, préoccupés, sages, etc. D'une autre part, le symbole s'étend pour faire une sorte d'un zoom out qui découvre la société entière: la belle rose, symbole de l'innocence, en face des baobabs, symbole du matérialisme, cette force destructrice de toute beauté. (BORGI, 2020, p. 10). Le symbole se complique aussi pour renvoyer à une image plus profonde: le serpent, symbole porteur de la mort et de la vie en même temps, désigne l'image circulaire de la naissance qui se termine par la mort, et puis recommence une autre naissance après la mort. L'auteur montrant le serpent soit comme un "anneau couleur de lune" (EXUPERY, 2003, p. 59), ou un "bracelet d'or" (EXUPERY, 2003, p. 60) ou aussi se laissant couler dans le sable comme "un jet d'eau" (EXUPERY, 2003, p. 84); il reflète cette ancienne image de la vie circulée: le venin du serpent, menant à la mort, il ouvre quand même une porte à la vie éternelle. Cela s'avère de la conversation du serpent avec le petit prince quand il lui a dit: "celui que je touche, je le rends à la terre dont il est sorti". (EXUPERY, 2003, p. 60)

A ce propos, Anne-Isabelle Mourier cite le sens du serpent en arabe, celui qui ne se diffère pas beaucoup du sens de la vie, puisque hayyah (serpent) et hayat (vie) ont les mêmes origines. Tandis que Al-Hay, l'un des principaux noms divins, "doit se 
traduire non par le vivant, comme on le fait souvent, mais par le vivifiant, celui qui donne la vie ou qui est le principe même de la vie". (MOURIER, 2001, p. 52)

C'est en résumant le voyage du petit prince, on découvrira que "d'étoile en étoile, de découverte en découverte, les pérégrinations du personnage éponyme sont autant de voyages initiatiques qui rattachent ce récit poétique à la tradition de la fable philosophique". (BEAUMARCHAIS, 1994, pp. 1512-1513).

- Intertextualité

Une lecture ordinaire du Petit prince révèle une série d'ouvrages sous-jacents entre les lignes du conte. Les Caractères de La Bruyère se dévoilent lucidement à travers les personnages avec leurs qualités personnelles: le roi, le vaniteux, le businessman, le buveur et autres, ne sont qu'un miroir de l'âme humaine avec tous ses défauts, ses déceptions et ses ambitions. D'un angle différent, on se trouve devant un conte ressemblant aux Fables de La Fontaine: les animaux et les plantes qui parlent, pensent et décident, ils rappellent un héritage très ancien dans l'histoire littéraire. On constate une sorte d'intertexte interne représenté dans le personnage principal, le petit prince, qui n'est rien que l'enfant merveille dans l'ouvrage de St. Exupéry Terre des hommes.

\section{- Illustration}

À l'exception de quelques images parsemées çà et là, l'ouvrage semble pauvre en images. Tandis que la littérature destinée à 
l'enfant devrait en fourmiller. Mais certains critiques voient que l'image peut enfermer l'imagination de l'enfant. Alain Vergnioux, en citant Bruno Duborgel, parle d'un "sevrage de l'imaginaire"! (VERGNIOUX, 2010, p. 43)

Cela traduit la tendance, dans les écrits destinés aux enfants, de présenter une image neutre, puisque "la bonne image est définitionnelle, explicative, descriptive. Elle fait corps avec une pédagogie de l'observation, de l'analyse et du classement". (VERGNIOUX, 2010, p. 43). Antoine de St. Exupéry avait dessiné les illustrations de son ouvrage. Même si la motivation derrière cette action n'est pas connue, mais nous pouvons justifier ce choix par une intention d'inciter le lecteur, quoiqu'il soit, à relâcher son imaginaire et non pas le freiner:

Le serpent boa: il semble d'une part un dessin quasi imaginaire, d'une autre part véridique. L'encyclopédie Larousse en constate cette vérité: "la taille de ces reptiles et leur mode de chasse sont en partie à l'origine de la terreur quasi sacrée qu'ils inspirent. En réalité, les boas constricteurs, dont l'origine remonte à une époque précédant la séparation des continents terrestres, ne sont pas très dangereux, même si certains d'entre eux sont les plus grands serpents du monde". (Larousse, 2020) Le fait que les boas avalent leurs proies est véritable. Ils s'enroulent autour des proies pour les étouffer. Ils peuvent rester des mois sans nourriture, et les boas en bonne santé peuvent rester à jeun pour plus d'une année si leurs proies sont peu grandes. Mais si nous avons à parler des espèces 
de proies favorites pour les boas, on en distingue des oiseaux, des opossums, des mangoustes, des rats, des écureuils, des agoutis, des pacas, des ocelots, voire des lapins. En ce qui concerne les proies les plus grandes, comme les caïmans, les cervidés, les pécaris, les agoutis, les pacas, les oiseaux, les tortues et beaucoup d'animaux aquatiques; elles sont les plus préférées pour les anacondas ou les boas constricteurs. Ceux-ci sont aussi amateurs de canards, de moutons, de chiens ou d'autres animaux domestiques qu'[ils parviennent] à capturer. (Larousse, 2020)

Le dessin de St. Exupéry s'approche de la vérité du boa, ce grand serpent avalant les fauves, mais cette vérité semble exagérée, parce que le boa avale cette fois un éléphant, et par la suite il prend sa forme. Le dessin de St. Exupéry dépasse les bords de la réalité: non pour le fait de l'avalement de l'éléphant, mais pour l'imaginaire dont il a lâché les brides. L'auteur compte sur l'imagination enfantine; et les enfants d'aujourd'hui ne sont pas fiables, à propos de l'imagination, à cause de l'enseignement matériel qu'ils ont reçu. Ils apprennent la mathématique, la géographie, le calcul, la physique, etc. Mais ils ne savent rien sur le rêve, pas beaucoup sur le dessin, ni sur l'espoir ou le sens du bonheur. Ces sentiments abstraits, ils n'apprennent pas à y réfléchir ni à en comprendre l'essence. A travers le dessin de l'écrivain, il a passé au lecteur enfant ainsi qu'au lecteur adulte une petite devinette: de quoi s'agit-il? Le narrateur, quand il était enfant, avait imaginé la scène de l'attaque contre l'éléphant de la 
part du boa. Il a sauté au résultat en dessinant la forme finale de ce drame, c'est-à-dire le serpent en forme d'éléphant sans détails physiques visibles. Le défi lancé de la part de l'écrivain est d'examiner l'innocence et la richesse d'imagination chez les lecteurs comme il l'a fait avec les personnes de son enfance. Si le lecteur, soit enfant ou adulte, réussit à voir ce qui est caché, s'il réussit à voir le dessin avec tous les détails invisibles, donc il réussira à l'examen existentiel, le secret enfantin qui les isole de tout le matérialisme destructif et qui leur assure le bonheur serein supérieur aux rancunes humaines et aux compétitions matérialistes.

Le dessin se transforme en chapeau, juste aux yeux des "grandes personnes", lorsque le petit prince, dernier représentant de la race des innocents, le voit comme l'a vu l'écrivain à son enfance, un boa avalant un éléphant.

Le boa au début du conte se montre serrant un fauve, ouvrant sa bouche pour l'avaler. Une image intimidante, mais impressionnante. Encore une fois à la fin du conte, le serpent apparait pendant la rencontre avec le petit prince, leur conversation ensemble, et puis la résignation du petit prince à la morsure du serpent pour retourner à sa planète. Cette morsure portait le salut du petit prince et la seule sortie du monde terrain au monde céleste, sa planète natale. L'illustration du serpent au deuxième cas n'est pas pareil à la première: cette fois le serpent 
semble paisible et familier. Son nœud connu est simple et nonmenaçant.

Le chapeau: se dévoile si simple du point de vue adulte; un dessin clair et matériel, aucune imagination n'est requise pour le comprendre. En dépit de cela, l'écrivain-dessinateur en donne des clés secrètes pour que les adultes parviennent à en décortiquer l'invisible. D'une part, les bordures du chapeau ne sont pas uniformes, une partie est plus allongée que l'autre, le haut est turgide d'un côté et presque plat de l'autre. Un dessin pareil devrait inciter l'imaginaire des personnes ayant du sens, mais la peinture semble aussi illogique du point de vue matérialiste. C'est presque un chapeau; les "grandes personnes" ne voient pas plus loin, tandis que le petit prince, comme l'enfant qu'était l'auteur auparavant, voit l'invisible, comme si le dessin obscur n'est que transparent. C'est le pouvoir que donne l'innocence à la raison enfantine.

Le petit prince: est un garçon aux cheveux dorés, scintillant, habillé d'une redingote colorée en rose et bleu ciel, des couleurs rêveuses et romantiques qui reflètent sa personnalité sensible. Ce type d'enfant est obsédant dans les écritures de St. Exupéry. Dans son ouvrage notoire Terre des hommes il voit dans le visage d'un enfant polonais pauvre qu'il rencontre, par hasard, dans le train, celui d'un enfant que "les petits princes des légendes n'étaient point différents de lui". (EXUPERY, Terre des hommes, 1939, p. 181) Dans plusieurs endroits ce petit prince se montre habillé 
différemment, mais l'habit exemplaire était celui de la redingote. Sur sa planète où il arrosait sa fleur unique il était habillé ordinairement d'une chemise et d'un pantalon avec une longue écharpe autour de son cou. Une apparence très simple mais romantique et rêveuse, rappelant celle des jeunes chanteurs et artistes aux années soixante-dix. Ses cheveux dorés semblent la plupart du temps comme ondulés et flottants dans l'air. Rien n'est plus romantique et sensible que cela!

Le turc: l'astronome turc avait deux apparitions contradictoires dans le conte; la première ridiculisée, la deuxième solennelle. La première apparence avec l'habit turc tout différent de celui européen dont il est habillé plus tard. Le laps temporel entre les deux apparences explique la frivolité des adultes qui ont refusé ses importantes découvertes à cause de ses vêtements différents des leurs, et quand il a changé d'habit et s'est habillé à l'européenne, ils l'ont accueilli avec applaudissements et il leur était bienvenu!

Le reste des illustrations concernant les autres animaux et personnes porte beaucoup d'explications qui prouvent l'existence d'un sens profond derrière chacune d'elles. Mais les exemples précédents sont les plus remarquables parmi eux.

\section{Conclusion}

L'une des raisons incitant à faire cette recherche est la relecture du conte d'Antoine de St. Exupéry. Si l'on lit ce conte dans un âge donné, le fait de le relire sera d'une vision tout différente. Le plus 
qu'on lit "Le petit prince" le plus profond qu'on contemple son contenu. La capacité de restaurer des liens et de faire des analogies sera plus à l'aise pour un lecteur adulte que pour un lecteur enfant.

Le conte fourmille de messages philosophiques et humanitaires qu'un lecteur enfant serait inapte de discerner même avec plusieurs lectures. La plupart du plaisir de la lecture de ce conte réside dans le fait de le voir d'une vue caléidoscopique. Ce qui nous importe dans cette recherche est la potentialité de l'œuvre elle-même, et cela est l'axe autour duquel tourne cette approche.

Certains facteurs distinguant la littérature pour enfants nous semblent clairs à travers cet ouvrage. Mais aussi, si nous avons de le classer du point de vue esthétique, il serait plus convainquant de le classer comme littérature pour adultes, ou simplement de la littérature en général, sans aucune taxinomie.

Cet ouvrage nous a montré la possibilité de trouver dans un livre destiné aux enfants des dessins primitifs, du vocabulaire simple, de l'imaginaire, de la répétition et du message direct. Mais aussi, nous y avons trouvé de la profondeur, de la sagesse, de la philosophie et surtout de l'humanisme.

Plusieurs maximes nous s'avèrent éminents à chaque lecture. Le message principal était renvoyé dès le début: l'écrivainnarrateur nous a fait remarquer que les adultes ont oublié qu'ils étaient aussi enfants. Partant de cette vérité manquée, il a opposé deux mondes lointains l'un de l'autre et si différents l'un de l'autre, 
le monde adulte et le monde enfantin. Cela nous implique, comme lecteurs adultes, à lire le conte dont nous faisons partie, soit pour découvrir ce qui nous a manqué de notre passé enfantin, soit pour explorer un monde des merveilles où tout est possible. Les chapitres derniers du conte nous en témoignent: l'aviateur avait à peine fini son eau, il traversait le désert avec le petit prince à la recherche d'un puits. Cet épisode est révélateur de la correspondance entre le désert et la vie de chacun de nous, le puits que chacun cherche n'est que l'amour que nous trouvons dans les petits détails de notre vie. C'est bien le trésor ultime, que si nous ne trouvons pas, nous mourrons de soif et de sècheresse. L'écrivain nous a appris qu'"on ne voit bien qu'avec le cœur. L'essentiel est invisible pour les yeux". La maxime n'est pas gratuite: ce n'est pas habituel pour un enfant de déchiffrer un tel message; ce qui nous donne une autre preuve que le conte n'était pas uniquement destiné aux enfants. Il y a toujours des messages cachés que seuls les adultes peuvent saisir.

Somme toute, St. Exupéry a réussi à travers son Petit prince à prouver qu'un ouvrage littéraire pourrait dépasser les limites de l'âge et que les lecteurs, à part les enfants, recevraient les messages délivrés par l'auteur, chacun selon son arrière-plan et son aptitude de s'y approfondir selon l'apprentissage, la culture ou l'état d'âme dominant. 


\section{Bibliographie}

(2021, janvier 11 ). Récupéré de https://des-livres-pour-changer-devie.com/le-petit-prince/

BEAUMARCHAIS, J.-P. d. (1994). Dictionnaire des littératures de langue française (S-Z). In J.-P. d. BEAUMARCHAIS, Dictionnaire des littératures de langue française ( $\mathrm{S}-\mathrm{Z})$. Paris: Bordas.

BEAUMARCHAIS, J.-P. d. (1994). Dictionnaire des œuvres littéraires de langue française (K-P). In J.-P. d. BEAUMARCHAIS, Dictionnaire des œuvres littéraires de langue française (K-P) (pp. 1512-1513). Paris: Bordas.

BORGI, R. (2020). Rouba BORGI, Le https://www.academia.edu/2103519/Le_Petit_Prince_Antoine_de_Sai nt_Exup\%C3\%A9ry_Etude_et_Commentaire. Récupéré de Rouba BORGI, Le pehttps://www.academia.edu/2103519/Le_Petit_Prince_Antoine_de _Saint_Exup\%C3\%A9ry_Etude_et_Commentaire

DEMERS, D. (1998, printemps). "Plaidoyer pour la littérature de jeunesse". Québéc français(numéro 109), pp. pp. 28-30.

EXUPERY, A. d. (1939). Terre des hommes. In A. d. EXUPERY, Terre des hommes (p. 181). Gallimard.

EXUPERY, A. d. (2003). Le petit prince. Gallimard, coll. FOLIO Junior.

JOUVE, V. (2002, Septembre). "Littérature pour adultes, littérature pour enfants: à chacun son jeu". La revue des livres pour enfants (dossier intitulé: Littérature en question)(numéro 206), pp. 69-77. 
Larousse. (2020, septembre 9).

https $/ /$ www.larousse.fr/encyclopedie/vie-sauvage/boa/184833.

Récupéré de https//www.larousse.fr/encyclopedie/viesauvage/boa/184833. Consulté le 9 septembre 2020.

MOURIER, A.-I. (2001, été). "Le petit prince de Saint-Exupéry: du conte au mythe". Études littéraires, vol. 33(numéro 2), pp. 43-54.

Nombres. (2021, janvier 9). Récupéré de http $/ /$ www.biblegateway.com/passage/?search=Nombres $\% 2021 \% 3$ A9\&version=LSG

SAYED, A. G. (2018). Interculturalité éducative dans la littérature de jeunesse française et égyptienne (thèse de doctorat). 34. Minia: Université de Minia.

VERGNIOUX, A. (2010). La littérature de jeunesse à l'école: "Des fictions sur mesure". Revue Lettre de l'enfance et de l'adolescence( numéro 79), pp. 41- 46. 


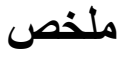

يتم التعرف على الحدود بين أدب الأطفال وأدب الكبار من قبل الأدباء وكذلك من قبل القراء. يحدث أحيانًا أن تتدمج هذه الحدود لتتتج نوعًا مختلطًا ينتمي على ما يبدو إلى أدب الطفل في الثكل، ولكنه يقترب بطريقة ما من الأدب العام، المعروف بأدب البالغين، من الجوهر .. أما بالنسبة لحكايته الأمير الصغير ، فقد كان سانت إكزوبيري أيضًا من قام برسم اللوحات المائية التي زينت الكتاب. الكاتب نفسه هو راوي القصة. لقد استوحى من مهنته كطيار حقائق معينة في الحكاية مثل وقوع الطائرة الذي كانت سبب الحكاية وبداية لقاءه مع الثخصية الرئيسية، الأمير الصغير • يأتي هذا الجزء ردا على حادثة حقيقية وقعت للمؤلف في قلب الصحراء بصحبة ميكانيكي في الثناثثنيات. قدم هذه الحكاية للسينما في نسخة الرسوم المتحركة من قبل مارك أوزبورن في عام 10 ـ ب. لا تدع إنسانية هذا العمل والفلسفة التي تكثر فيه أي شك في أن الحكاية مخصصة للقراء من جميع الأعمار • بناءً على هذا الافتراض، سنقوم بتحليل الجوانب المختلفة التي تجعل من هذا العمل العالمي تحفة فنية للأطفال والكبار على قدم المساواة. 